\title{
Data acquisition, parameter extraction and characterization of active components using integrated instrumentation system
}

\author{
H. Bourdoucen ${ }^{1}$ and A. Zitouni ${ }^{2}$ \\ ${ }^{I}$ Department of Electrical and Computer Engineering, College of Engineering, Sultan Qaboos University, \\ P. O. Box 33 Al-Khodh, Muscat, Oman 123 \\ ${ }^{2}$ University of Boumerdes, Department of Electrical Engineering, 35000, Algeria \\ E-mail: hadj@squ.edu.om
}

\begin{abstract}
A data acquisition, parameter extraction and characterization system for electronic active components is presented in this paper. High sensitivity measuring equipments were used for data acquisition and effective extraction models based on optimization techniques developed to obtain the parameters of $p-n$ junction diodes, Schottky diodes, field effect transistors and bipolar junction transistors. The performance of the developed extraction techniques are apparent via comparing experimental data with Spice simulated data using the model parameter that is graphically extracted and also those extracted using optimization techniques. The performance of the developed extraction techniques has been demonstrated by comparing the experimental characteristics with Spice simulated curves using default parameters and model parameters extracted using graphical and optimization techniques. The relative excursions of the simulated $I-V$ characteristics of most investigated devices were less than $2.5 \%$ with respect to the experimental curves, which shows the accuracy and effectiveness of the developed system. A number of software routines have also been implemented under Matlab environment to extract the Spice model parameters for different electronic devices.
\end{abstract}

Keywords: parameter extraction, Spice model, BJT, FET, p-n junction, Schottky diode.

Manuscript received 25.11.08; accepted for publication 02.03.09; published online 20.03.09.

\section{Introduction}

As the electronic components market continues to grow up and overcomes many barriers related to shrinking of devices geometry, the characterization tools used to access its various products' behavior need to advance at a similar trend. Within semiconductor devices field, many measuring instrument manufacturers have developed new sophisticated products and improved their equipments to get high measurement resolution and hence, allow precise semiconductor device parameters to be extracted [1-8]. Furthermore, most of these instruments are provided with wired and wireless interfaces to be remotely controlled by computers [9] and automate measurement and analysis operations. The Parallel and USB interface buses for instance, are carefully designed instrumentation interfacing means that simplify integrating measuring instruments and a computer into one system.

This work presents data acquisition, parameter extraction and characterization of electronic active components using an implemented integrated instrumentation system. This takes full advantage of advanced features of current measuring instruments that operate remotely via interface bus in order to characterize accurately a wide set of passive and active electronic components. These instruments are widely encountered in electronic industry as well as in applied research. The flow of data through the interface bus is completely monitored using developed integrated software. In addition to this, and in order to make the system full featured, a set of graphical based routines under Matlab environment have been implemented. These routines allow extraction of SPICE model parameters for a large number of electronic devices.

With regard to parameter extraction, traditionally, there are two different approaches that have been used for parameter extraction. First, a large number of local methods where the parameters are determined two by two as the slope and the intercept of a straight line [10] and the influence of other effects is often ignored. The second approach is based on the use of general optimization techniques [11] where all parameters (or subsets thereof) are extracted simultaneously, sometimes 
even using data from several different device sizes. These methods show good agreement between measured and simulated data but they are CPU-intensive and require relatively large number of data points [1]. As an alternative to these two approaches, least square fitting techniques on linearized model equations have been proposed [12]. Another alternative is the so-called direct parameter extraction using only a small number of data points. This approach was first introduced by Hamer [13] and was then developed further by several researchers $[2,14]$.

Parameter extraction methods developed in this work are mostly based on local methods and optimization approaches.

Extraction of the parameters by using the local methods (also known as graphical methods) depends on the equation forms on which the theory of the derived model is based. These methods are in no way considered to be general, since for each device model the resulting equations are different. In general, by judicious handling the model equations (sometimes using some assumptions) one ends up with a linear form. Parameters are then determined often two by two, by means of graphical methods as a slope and an intercept of a straight line. The slope and the intercept of the line are usually found using least square fitting techniques on a number of measured data points.

The main advantage of optimization methods is that the model parameters for new or modified models are easy to extract. This is because the optimizer can use the same modules as the circuit simulator to calculate the device terminal variables mainly currents and voltages. Hence, this guarantees that the same equations are used by the simulation and the extraction programs. All extraction algorithms based on optimization techniques try to minimize an error function that is a measure of the distance between measured and calculated data points. Even after the error function has been minimized, there will be a difference between measured and calculated data points, since the model always represents an approximation to reality, therefore and the data points contain measurement noise. The algorithm selected to build the parameters extraction programs using optimization methods is based on the gradient approach and more particularly on the well known LevenbergMarquardt algorithm [1].

\section{Experimental setup}

The whole measurement system shown in Fig. 1 is composed of hardware and software parts. The hardware parts is composed mainly of high performance and accurate Hewlett-Packard instruments that are provided with HPIB bus interface cards that make them remotely controlled by any IBM compatible computer. This allows automated monitoring, measurement, analysis and data presentation. Thus, many electrical characterisation techniques of both passive and active electronic components are feasible with reduced efforts.

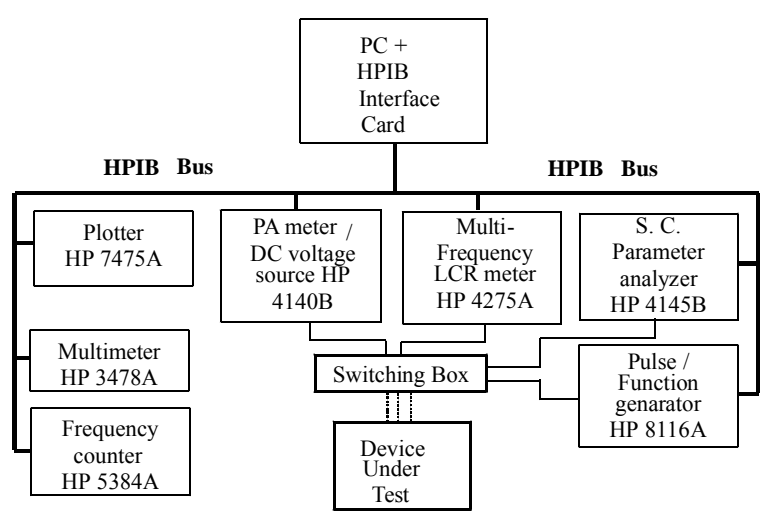

Fig. 1. Hardware configuration of the designed setup.

One of the main instruments in the measurement systems is the well known HP 4140B and HP 4145B. Other devices shown in the measurement diagram (Fig. 1) are used as complements for the functions not provided by both the HP 4275A and 4140B. For instance, The HP model 3478A digital multimeter can be used to get more precise measurements for the voltage values delivered by the HP $4140 \mathrm{~B}$, especially in low voltage ranges.

The system developed is fully monitored by an IBM compatible computer which allows the user to be able to perform all device settings and operations remotely from his computer desk, without acting on device front panels.

The HP 4140B is pico-ammeter that has a basic accuracy of $0.5 \%$ over wide measurement ranges $\left( \pm 0.001 \times 10^{-12}\right.$ to $\left.\pm 1.999 \times 10^{-2} \mathrm{~A}\right)$ enabling stable current measurement at $10^{-15} \mathrm{~A}$ ( \pm 1 count). Its DC voltage source has an output range of $\pm 100 \mathrm{~V}$ in $100 \mathrm{mV}$ steps or $\pm 10 \mathrm{~V}$ in $10 \mathrm{mV}$ steps and, the ramp rate can be set from $0.001 \mathrm{~V} / \mathrm{s}$ to $1 \mathrm{~V} / \mathrm{s}$ at $0.001 \mathrm{~V} / \mathrm{s}$ resolution. In addition each of its two DC voltage sources has a current limiter that can be set to $100 \mu \mathrm{A}, 1 \mathrm{~mA}$ or $10 \mathrm{~mA}$ to avoid damaging the device under test by excessive current. A key capability of the HP 4140B is its ability to make accurate current-voltage $(I-V)$ and capacitancevoltage $(C-V)$ measurements, which makes it an excellent characterisation tool in the microelectronics area.

The HP 4275A multi-frequency LCR meter is a high performance, fully automatic test instrument designed to measure the various component parameter values of an impedance element in the relatively high frequency region $(10 \mathrm{kHz}$ to $10 \mathrm{MHz}$ ). The instrument is able to measure inductance, capacitance, resistance, dissipation factor, quality factor, conductance, susceptance, and reactance values. In addition to this, it can determine the absolute value of the vector impedance as well as its phase angle, over a wide range with high accuracy and speed. The range of measurement capabilities of the HP 4275A is enhanced by the 10 spot test frequencies, which have the accuracy 
$0.01 \%$ and selectable from $10 \mathrm{kHz}$ to $10 \mathrm{MHz}$ in a $1-2-$ 4-10 sequence. The measuring range for capacitance is from $0.001 \mathrm{fF}$ to $199.99 \mu \mathrm{F}$, from $0.01 \mathrm{nH}$ to $199.99 \mathrm{H}$ for inductance, it is from $0.01 \mathrm{mOhm}$ to $19.999 \mathrm{MOhm}$ for resistance and impedance. Its accuracy is within the ranges of 0.1 to $5 \%$, depending on the test signal level and operating frequency.

A number of other HP instruments such as the HP $8116 \mathrm{~A}$ pulse/function generator, the HP 5384A frequency counter, HP 3478A digital multimeter, which are very powerful bench instruments that are able to perform very well whether in a research laboratory or within a industrial production area. Added to the above accurate instruments, is the well known HP 4145B Semiconductor Parameter Analyser that is fully automatic, high performance, programmable test instrument designed to measure, analyse, and graphically display the characteristics of a wide range of semiconductor devices such as diodes, bipolar transistors, field-effect transistors, semiconductor wafers, integrated circuits, and other devices. A switching box is used to make the necessary connections for two, three or four terminals of the devices under test.

The software part of the developed integrated system has been developed using two main principal files, the control file and the Data file.

The control file is used as a "pipe", through which the ASCII character string commands are sent to the HP equipment. It is by this mean that each measuring instrument, when addressed, can process commands that are sent to it through the bus. In addition, the integrated software exploits the intelligence of HP instruments to a great extent by using the TRIGGER mode feature. Indeed, many HP instruments, such as the HP 4140B, have the capability to be pre-programmed in order to see them perform their individual actions simultaneously or

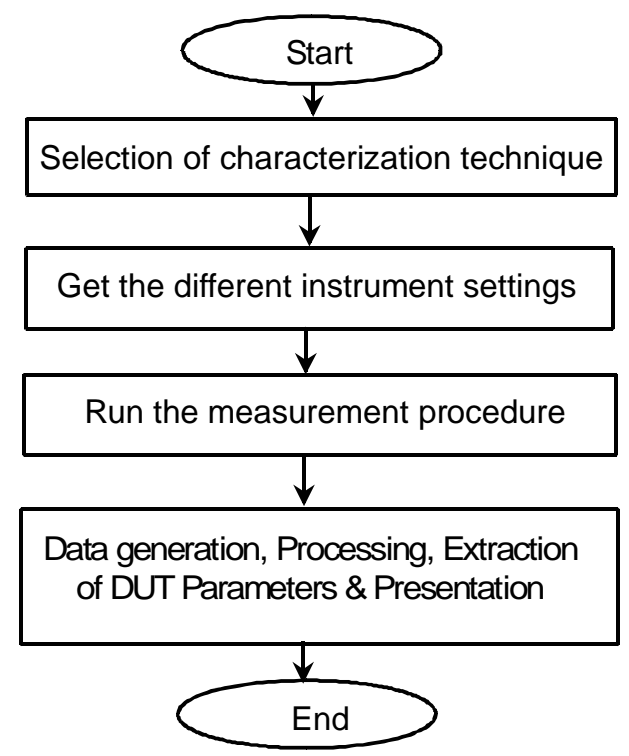

Fig. 2. The block diagram of the integrated software sequence. in sequence, depending on the current application. The control file can also operate as an output file to read the bus status string and the bus errors may thus be detected. The second file, data file, is used to collect device measurements to be processed by the controller. Each device is able to produce an ASCII string as formatted output data that contains measurement information such as data status (normal data, overflow, underflow, ...), measurement function (current-voltage measurements, capacitance-voltage measurements, capacitance with dissipation factor measurements, ...) and so on. The output data needs then to be filtered to get meaningful outcomes. The software's run time is presented in Fig. 2.

\section{Methods of parameter extraction}

In addition to the developed software that monitors and controls the instruments, and collects data, another program has been developed to include some extra useful utilities that allow portability of data files to be processed by MATLAB, where they can be processed to give useful components' parameters in an easy and faster way. The combination of the above hardware and software components developed allows to get accurate and trustful data for all the devices characterized.

\subsection{Extraction of diode model parameters}

\subsubsection{Static model parameters measurement}

The model used by SPICE program to approximate the $\mathrm{p}$-n junction or a Schottky diode needs four parameters specified by the forward mode as IS $\left(I_{S}\right)$ (saturation current), $\mathrm{N}(n)$ (emission coefficient in the reverse mode), BV $\left(V_{B}\right)$ (breakdown voltage), IBV $\left(I_{B}\right)$ (breakdown current) and GMIN $(G)$ (reverse conductance just before breakdown).

To extract the forward mode parameters, the plot of $\log \left(I_{d}\right)$ with respect to $V_{d}$ is used ( $I_{d}$ and $V_{d}$ are the current and voltage of the diode). This plot shows a linear region where the relation $\log \left(I_{d}\right)=\log \left(I_{S}\right)+\frac{1}{n V_{T}} V_{d}$ is valid, $V_{T}$ being the thermal voltage of the junction. Hence, fitting $\log \left(I_{d}\right)$ with respect to $V_{d}$ gives the value of both $n$ and $I_{S}$ in a straight forward way. If we let $\log \left(I_{d}\right)=a_{1} V_{d}+a_{2}$, where $a_{1}$ and $a_{2}$ are constants, then

$n=\frac{1}{a_{1} V_{T}}$

and

$$
I_{S}=e^{a_{2}} .
$$

To extract the parameter $G$ that is the reverse conductance, the slope of $I_{d}$ with respect to $V_{d}$ in the reverse mode, just before the breakdown, is calculated. The breakdown voltage and current parameters can also be calculated from curve fitting in the breakdown region. 


\subsubsection{Dynamic model parameters measurement}

In both large and small-signal dynamic models, the main parameters that are necessary to know are: $\tau_{D}$ (transit time of the diode), $\phi_{0}$ (diode built-in voltage), $C_{d}(0)$ (zero-bias depletion capacitance) and $m$ (coefficient used to calculate the depletion capacitance). $C_{d}(0)$ is obtained from measurements of the junction capacitance at zero bias condition. Then $\phi_{0}$ and $m$ are extracted using optimization methods.

\subsection{Extraction of JFET model parameters}

The model used to approximate the electric behavior of the junction field effect transistor (JFET) needs eleven (11) parameters that are: $C_{G D}(0)$ and $C_{G S}(0)$, (zero-bias gate-drain and gate-source capacitances), $m$ (grading coefficient), $\phi$ (built-in voltage), $I_{S}$ (saturation current), $n$ (emission coefficient), $\beta$ (transconductance coefficient), $V_{T 0}$ (threshold voltage), $\lambda$ (channel-length modulation), $r_{S}$ and $r_{D}$ (source and drain ohmic resistances). These are expressed in the following equations.

$$
I_{D}=\left\{\begin{array}{l}
0 \quad \text { for } V_{G S}-V_{T 0} \leq 0 \\
\beta\left(V_{G S}-V_{Y 0}\right)^{2}\left(1+\lambda V_{D S}\right) \text { for } 0<\mathrm{V}_{\mathrm{GS}}-V_{T 0} \leq V_{D S} \\
\beta V_{D S}\left[2\left(V_{G S}-V_{T 0}\right)-V_{D S}\right]\left(1+\lambda V_{D S}\right) \\
\quad \text { for } 0<V_{D S}<V_{G S}-V_{T 0}
\end{array}\right.
$$

For the equation of the drain currents (3), three parameters $\beta, V_{T 0}$ and $\lambda$ are required. The currents $I_{G D}$ and $I_{G S}$ are expressed as

$$
\begin{gathered}
I_{G D}=\left\{\begin{array}{cc}
-I_{S} & \text { for } V_{G D} \leq-5 n V_{T} \\
I_{S}\left(e^{\frac{V_{G D}}{n V_{T}}}-1\right. & \text { for } \mathrm{V}_{\mathrm{GD}}>-5 n V_{T}
\end{array}\right. \\
I_{G S}=\left\{\begin{array}{cc}
-I_{S} & \text { for } V_{G S} \leq-5 n V_{T} \\
I_{S}\left(e^{\frac{V_{G S}}{n V_{T}}}-1\right. & \text { for } \mathrm{V}_{\mathrm{GS}}>-5 n V_{T}
\end{array}\right.
\end{gathered}
$$

For the above equations related to gate-drain and gate-source junctions (4), (5), two parameters $\left(I_{S}\right.$ and $n$ ) should be known.

The equations (6) below may be used in approximating the large signal behavior of the gate-drain and the gate-source junctions. In this case, four parameters $m, \phi, C_{G D}(0)$ and $C_{G S}(0)$ are needed.

$C_{D}=\frac{d Q_{D}}{d V_{D}}= \begin{cases}\tau_{D} \frac{d I_{D}}{d V_{D}}+C_{d}(0)\left(1-\frac{V_{D}}{\phi_{0}}\right)^{-m} & \text { for } V_{d}<F C \times \phi_{0} \\ \tau_{D} \frac{d I_{D}}{d V_{D}}+\frac{C_{d}(0)}{F_{2}}\left(F_{3}+\frac{m V_{D}}{\phi_{0}}\right) & \text { for } V_{d} \geq F C \times \phi_{0}\end{cases}$

In addition to the above parameters, the drain and source ohmic series resistances $\left(r_{D}\right.$ and $\left.r_{S}\right)$ can be added.

In the section below, the extraction methods of these parameters will be developed. Note that the method of extraction of the parameters related to the two gate-drain and gate-source p-n junctions, are the same as those used to extract $\mathrm{p}-\mathrm{n}$ junction model parameters.

1) Extraction of $C_{G D}(0)$ and $C_{G S}(0)$ :

These two parameters are obtained by direct measurement at zero bias of the gate-drain and gatesource junctions capacitance using the HP 4275A model LCR meter at a given frequency value and oscillator voltage level.

2) Extraction of $m$ and $\phi$ :

They are obtained as for the p-n junction diode, using the optimization method on the equation of the depletion capacitance with respect to the applied reverse voltage. Since SPICE program uses the same parameters for both junctions, then the average values of extracted parameters from the two junctions are considered.

3) Extraction of $I_{S}$ and $n$ :

The same technique used for the p-n junction diode is used, and as it is the case for $m$ and $\phi$ parameters, average values of extracted parameters from both junctions are considered.

4) Extraction of $\beta$ and $V_{T 0}$ :

$\beta$ and $V_{T 0}$ are obtained at the same time from the curve of $\sqrt{I_{D}}$ with respect to $V_{G S}$ for a large enough value of $V_{D S}$ to ensure the transistor operating in the saturation region. Indeed, in this region we can consider that the drain current is:

$I_{D} \approx \beta\left(V_{G S}-V_{T 0}\right)^{2}$

and hence,

$\sqrt{I_{D}} \approx \sqrt{\beta}\left(V_{G S}-V_{T 0}\right)$

Therefore, fitting $\sqrt{I_{D}}$ with respect to $V_{G S}$ by a straight line gives $\beta$ as the square value of the obtained curve slope and $V_{T 0}$ as the intercept with the $V_{G S}$ axis.

5) Extraction of $\lambda$ :

The extraction of $\lambda$ requires the measurement of the output conductance of the device in the saturation region for a given value of $V_{G S}$. This is shown by considering the definition of the output conductance as follows: $g_{0}=\frac{d I_{D}}{d V_{D S}}=\beta\left(V_{G S}-V_{T 0}\right)^{2} \lambda \approx \lambda I_{D}$.

Thus, by measuring $g_{0}$ at different values of $I_{D}$, one may estimate the value of $\lambda$ as the slope of the curve of $g_{0}$ versus $I_{D}$ as shown in Fig. 3 .

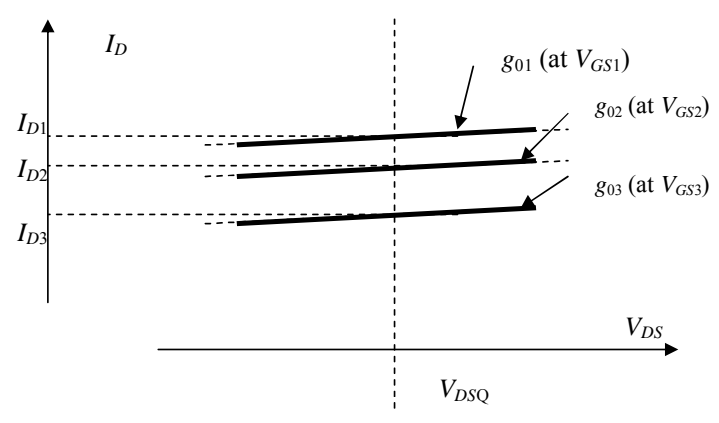

Fig. 3. Extraction of the variable $\lambda$. 


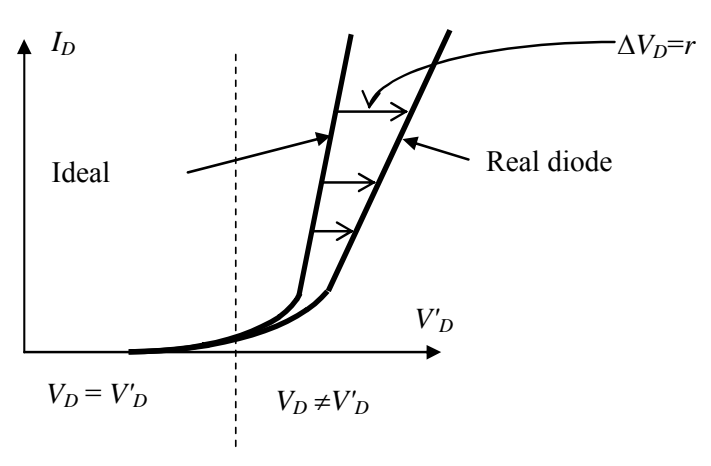

Fig. 4. Extraction of the drain and source series resistances.

6) Extraction of $r_{S}$ and $r_{D}$ :

From the static model of the junction diode, it is clear that the effect of the series resistances $r_{S}$ and $r_{D}$ is to shift the ideal characteristics $I_{D}=f\left(V_{D}\right)$ by an amount of $\Delta V_{D}=r I_{D}$ as shown in Fig. 4.

Here, $r$ stands for either $r_{S}$ or $r_{D}$ depending on the considered junction, and can be extracted using the following equation $r=\frac{\Delta V_{D}}{I_{D}}$.

In practice, several values are calculated and the average value is adopted.

Note that the extraction of the series resistances in the case of JFET devices is possible because of their relatively high values in comparison to junction diodes. In practice, we have noticed that the effect of the series resistances of the JFET becomes significant before the maximum current limit of the HP 4145B $(100 \mathrm{~mA})$ is reached. This is neither the case for the $\mathrm{p}-\mathrm{n}$ junction nor for the Schottky diode.

\subsection{Extraction of BJT model parameters}

The Gummel-Poon model parameters [17] can be obtained for their majority by investigating the curves of $\log \left(I_{C}\right)$ and $\log \left(I_{B}\right)$ with respect to $V_{B E}$ in both normal and reverse operating modes for a given value of $V_{B C}[15]$.

The Gummel-Poon model equations are then obtained in the different operating regions as per the following expressions:

Normal active

$$
I_{C}=\frac{I_{S}}{q_{b}}\left(e^{\frac{V_{B E}}{n_{F} V_{T}}}+\frac{q_{b}}{\beta_{R}}\right)+C_{4} I_{S}
$$

region:

$V_{B E}>-5 n_{F} V_{T}$

and

$$
I_{B}=I_{S}\left[\frac{1}{\beta_{F}}\left(e^{\frac{V_{B E}}{n_{F} V_{T}}}-1\right)-\frac{1}{\beta_{R}}\right]+
$$

$V_{B C} \leq-5 n_{R} V_{T}$

$$
C_{2} I_{S}\left(e^{\frac{V_{B E}}{n_{E L} V_{T}}}-1\right)-C_{4} I_{S}
$$

$$
\begin{aligned}
& I_{C}=-\frac{I_{S}}{q_{b}}\left[e^{\frac{V_{B C}}{n_{R} V_{T}}}+\frac{q_{b}}{\beta_{R}}\left(e^{\frac{V_{B C}}{n_{R} V_{T}}}-1\right)\right]-
\end{aligned}
$$

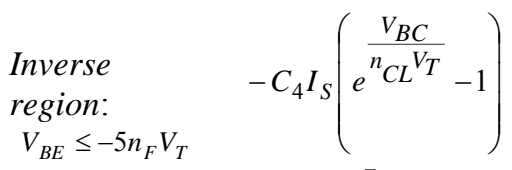

$$
\begin{aligned}
& \begin{aligned}
V_{B C}>-5 n_{R} V_{T} & I_{B}=-I_{S}\left[\frac{1}{\beta_{F}}-\frac{1}{\beta_{R}}\left(e^{\frac{V_{B C}}{n_{R} V_{T}}}-1\right)\right. \\
& -C_{2} I_{S}+C_{4} I_{S}\left(e^{\frac{V_{B C}}{n_{C L} V_{T}}}-1\right)
\end{aligned} \\
& I_{C}=\frac{I_{S}}{q_{b}}\left[\left(e^{\frac{V_{B E}}{n_{F} V_{T}}}-e^{\frac{V_{B C}}{n_{R} V_{T}}}\right)-\frac{q_{b}}{\beta_{R}}\left(e^{\frac{V_{B C}}{n_{R} V_{T}}}-1\right)\right]- \\
& -C_{4} I_{S}\left(e^{\frac{V_{B C}}{n_{B C} L_{T}}}-1\right) \\
& \begin{array}{l}
\text { Saturated } \\
\text { region: }
\end{array} \\
& V_{B E}>-5 n_{F} V_{T} \\
& \text { and } \\
& V_{B C}>-5 n_{R} V_{T} \\
& I_{B}=I_{S}\left[\frac{1}{\beta_{F}}\left(e^{\frac{V_{B E}}{n_{F} V_{T}}}-1\right)+\frac{1}{\beta_{R}}\left(e^{\frac{V_{B C}}{n_{R} V_{T}}}-1\right)\right]+ \\
& +C_{2} I_{S}\left(e^{\frac{V_{B E}}{n_{E L} V_{T}}}-1\right)+C_{4} I_{S}\left(e^{\frac{V_{B C}}{n_{C L} V_{T}}}-1\right) \\
& \underset{V_{B E} \leq-5 n_{F} V_{T}}{\text { Off region: }} \quad I_{C}=\frac{I_{S}}{\beta_{R}}+C_{4} I_{S} \\
& V_{B C} \leq-5 n_{R} V_{T} \quad I_{B}=-I_{S}\left(\frac{\beta_{F}+\beta_{R}}{\beta_{F} \beta_{R}}\right)-C_{2} I_{S}-C_{4} I_{S}
\end{aligned}
$$

In the normal mode, $C$ is in fact $C_{2}, I_{K}$ is $I_{K F}, n$ is $n_{F}, n_{L}$ is $n_{E L}$ and $\beta$ is $\beta_{F}$, while in the reverse mode (the collector and the emitter interchange their roles); $C$ is $C_{4}$, $I_{K}$ is $I_{K R}, n$ is $n_{R}, n_{L}$ is $n_{C L}$ and $\beta$ is $\beta_{R}$. In addition to these parameters, two other parameters also need to be extracted, they are respectively the early voltage in normal and reverse modes $\left(V_{A}\right.$ and $\left.V_{B}\right)$, which are used to approximate the currents $I_{C C}$ and $I_{E C}$.

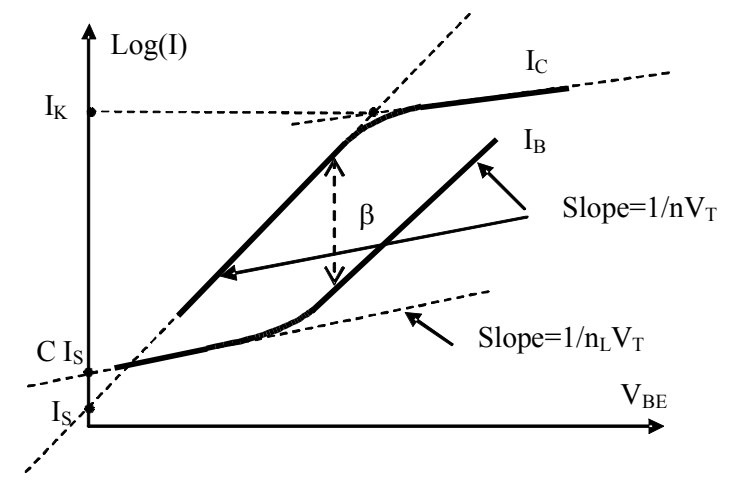

Fig. 5. Plot of $\log \left(I_{C}\right)$ and $\log \left(I_{B}\right)$ versus $V_{B E}$. 
7) Extraction of $I_{S}$ and $n$ :

With regard to the curve of $I_{C}$, by extrapolating through the region with the slope $1 / n V_{T}$, the value of the transistor saturation current $I_{S}$ is obtained. On the other hand, the emission factor $n$ is obtained from the curve slope as shown previously for the $\mathrm{p}-\mathrm{n}$ junction diode.

8) Extraction of $I_{K}$ :

$I_{K}$ is the knee current; it models the drop in $\beta$ at high collector currents due to high-level injection. If we assume that low-level and high-level injection effects could be distinguished by regions, a value of $I_{K}$ could be obtained by the intersection of the two asymptotes as shown in Fig. 5.

9) Extraction of $C$ and $n_{L}$ :

With regard to the curve of $I_{B}$, by extrapolating the region with the slope $1 / n_{L} V_{T}$ at low current levels, the value of $C$ can be determined and, by determining this slope, $n_{L}$ can be found. These parameters describe the non-ideal component of $I_{B}$ which is dominant at low currents. This component is responsible for the drop in $\beta$ at low currents.

10) Extraction of $\beta$ :

$\beta$ is the maximum value of the ratio $I_{C}$ to $I_{B}$ when the transistor is in the normal active region. $\beta$ can be determined by the regions of the two curves where they are parallel. In this region, both $I_{C}$ and $\mathrm{I}_{\mathrm{B}}$ have dominant ideal components, and hence $I_{C}$ can be considered to be proportional to $I_{B}$.

11) Extraction of $V_{A}$ and $V_{B}$ :

$V_{A}$ (or $V_{B}$ in the reverse mode) is the early voltage that models the effect on the transistor characteristics of the base-width modulation.

The simplest method of obtaining $V_{A}$ is from the slope of $I_{C}$ versus $V_{C E}$ curve in the linear region. The base voltage should be constant and the transistor should be biased to its normal operating point. In the forward mode, the collector current can be simplified as:

$I_{C}=\frac{I_{S}}{q_{b}} e^{\frac{V_{B E}}{n_{F} V_{T}}}$

Therefore, by keeping the base voltage constant, the collector current would be proportional to $1 / q_{b}$ and, if $V_{B E}$ is small enough so that $V_{C E}$ is approximately equal to $V_{B C}$, we will end up with the following result [15]:

$I_{C} \approx \frac{I_{S}}{q_{1}} e^{\frac{V_{B E}}{n_{F} V_{T}}}=I_{S} e^{\frac{V_{B E}}{n_{F} V_{T}}}\left(1+\frac{V_{C E}}{V_{A}}\right)$.

It is best to repeat the extraction at few base-emitter voltages and average the obtained $V_{A}$.

In the previous method, to determine $V_{A}$ it has been assumed the voltage $V_{B C}$ small enough so that $q_{b}$ is dependent only on $V_{A}$ and $V_{B E}$. This is why in practice the obtained values of $V_{A}$ for different values of $V_{B E}$ are often very different.

A more accurate method to determine $V_{A}$ and $V_{B}$ at the same time is, the use of the curve $\log \left(I_{C}\right)$ versus $V_{B E}$ (and $\log \left(I_{E}\right)$ versus $V_{B C}$ ) in both normal and reverse modes, for two distinct values of $V_{B C}\left(V_{B E}\right)$. For a given value $V_{B E 0}$ of $V_{B E}$, one can obtain the following equation in the normal mode:

$\frac{I_{C}\left(V_{B C}=0\right)}{I_{C}\left(V_{B C}=V_{B C 1}\right)}=\frac{1+\frac{V_{B E 0}}{V_{B}}+\frac{V_{B C 1}}{V_{A}}}{1+\frac{V_{B E 0}}{V_{B}}}$.

And for a given value $V_{B C 0}$ of $V_{B C}$, in the reverse mode:

$\frac{I_{E}\left(V_{B E}=0\right)}{I_{E}\left(V_{B E}=V_{B E 1}\right)}=\frac{1+\frac{V_{B C 0}}{V_{A}}+\frac{V_{B E 1}}{V_{B}}}{1+\frac{V_{B C 0}}{V_{A}}}$. and $V_{B}$.

Equations (17) and (18) can be solved to get $V_{A}$

\section{Experimental data acquisition and parameter extraction}

\subsection{Testing p-n junction and Schottky diodes}

In the following section, results extracted from the $\mathrm{p}-\mathrm{n}$ junction diode 1N4003 and the Schottky ITS 7818 model diodes will be presented.

These are obtained from measurements of the forward and reverse diodes DC characteristics, and junction depletion capacitances at ambient temperature (around $25^{\circ} \mathrm{C}$ ).

The obtained model parameters in the forward biasing conditions can be considered very good as indicated in Figs 6 and 7. However, in the reverse biasing conditions the Schottky diode fits better the theoretical model as shown in Fig. 8. The summarized results are presented in Table 1. The results shown in this table agree with the expected ones. For example, practical values of the emission coefficient for $p$-n junction diodes are between 1 and 2, while it is close to 1 for Schottky diodes [15].

The same table shows the extracted parameters related to the depletion capacitance of the large signal model.

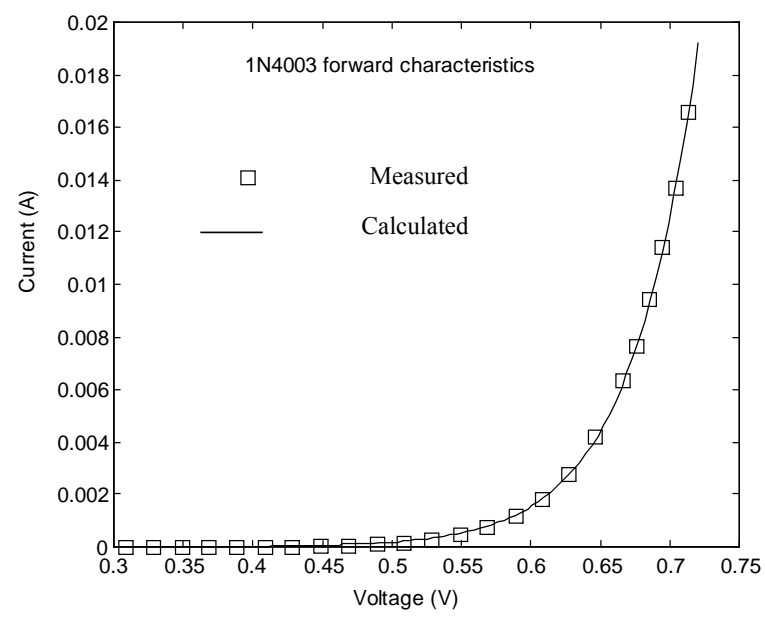

Fig. 6. DC model characteristics for the 1N4003 diode. 
Table 1. Extracted parameters of both p-n junction and Schottky diodes.

\begin{tabular}{cccccc} 
Device & $I_{S}(\mathrm{~A})$ & $\mathrm{N}$ & $C_{D}(0)(\mathrm{pF})$ & $\phi(\mathrm{V})$ & $\mathrm{M}$ \\
\hline 1N4003 & $5.32 \times 10^{-9}$ & 1.86 & 42.26 & 0.432 & 0.452 \\
ITS & $1.27 \times 10^{-6}$ & 0.9985 & 428.5 & 0.382 & 0.463 \\
5818 & & & & & \\
\hline
\end{tabular}

As it is indicated in Fig. 9, the model for the junction capacitance curves fit very well the experimental data for both the frequencies used, i.e. $10 \mathrm{kHz}$ and $1 \mathrm{MHz}$.

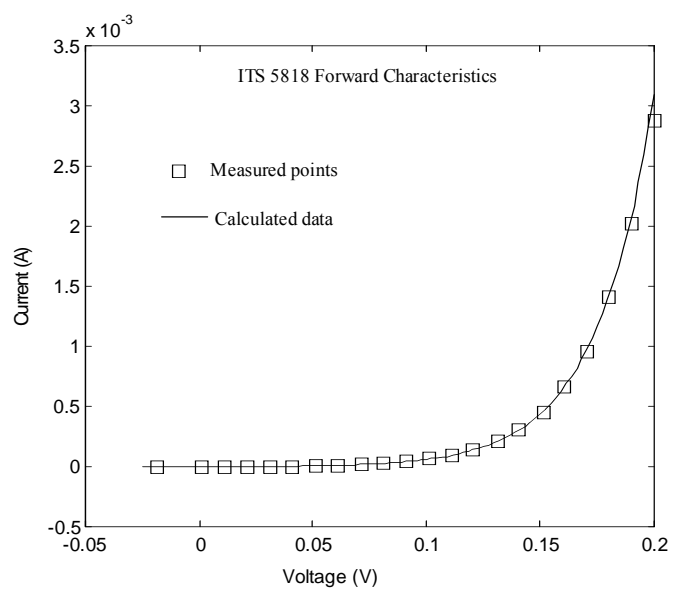

Fig. 7. DC model characteristics for ITS5818 Schottky diode.

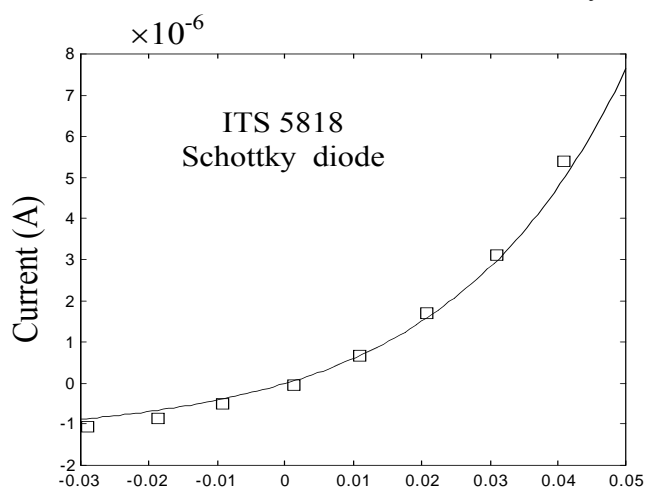

Applied voltage (V)

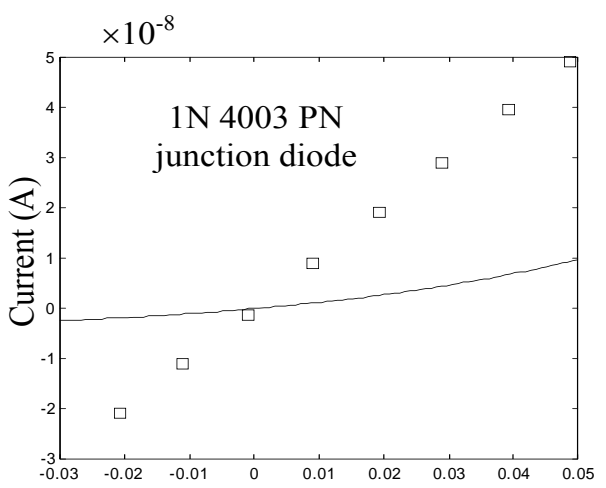

Applied voltage (V)

Fig. 8. p-n junction and Schottky diodes in reverse biasing modes. Squares: measured points; solid lines: calculated data.

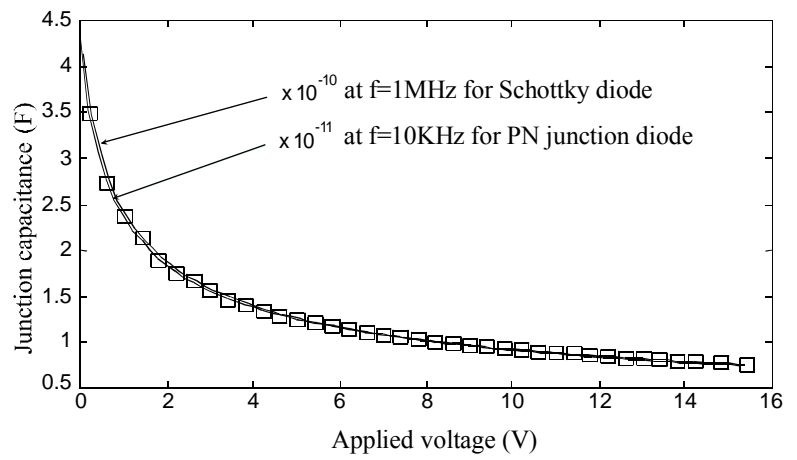

Fig. 9. Depletion capacitance as a function of applied reverse voltage for both a $\mathrm{p}-\mathrm{n}$ junction diode and Schottky diode. Note that the capacitance values for the Schottky diode are about ten times less than the capacitance of the $p-n$ junction diode.

\subsection{Testing of Junction Field Effect Transistors (JFETs)}

In this section, the results extracted from experimental measurements of the JFET 2 N3823 are presented. In order to extract meaningful parameters for the device, measured data of drain current $I_{D}$ versus drain voltage $V_{D S}$ for several values of $V_{G S}$ are compared with simulation results using the ORCAD program with model default values. The obtained results are shown in Fig. 10.

As it can be noticed from this figure, the data obtained by simulation are considerably different from the measured ones, even if the model used is known to be very accurate. This example shows clearly that accurate models are not sufficient to obtain accurate simulated data.

The difference between simulated and experimental data in the above example can be explained by the fact that the simulation program uses default model parameters to perform numerical simulation. These parameters are often defined for the first time when the software is installed, and can be modified according to the user application. A list of some default parameters is presented in Table 2 [16].

The model parameters of the device have been calculated from measured data using methods of extraction presented above and they are also indicated in Table 2 . The simulation program has been launched but with the extracted parameters this time, the obtained results are shown in Figs 10 and 11. They show significant improvement in the simulation results. The relative excursion between the simulated $I-V$ characteristics with respect to the experimental curves might exceed $220 \%$ for $V_{G S}=0$ and $150 \%$ for $V_{G S}=-1$ for curves obtained using default values. However, these excursions are only limited to less than $1.2 \%$ for $V_{G S}=0 \mathrm{~V}$ and $0.5 \%$ for $V_{G S}=-1 \mathrm{~V}$ using the extracted values.

Hence, from this simple example one can deduce that the results obtained from simulation are more accurate when actual model parameters are used, and that the developed methods of extraction of JFET parameters model described here are effective. 


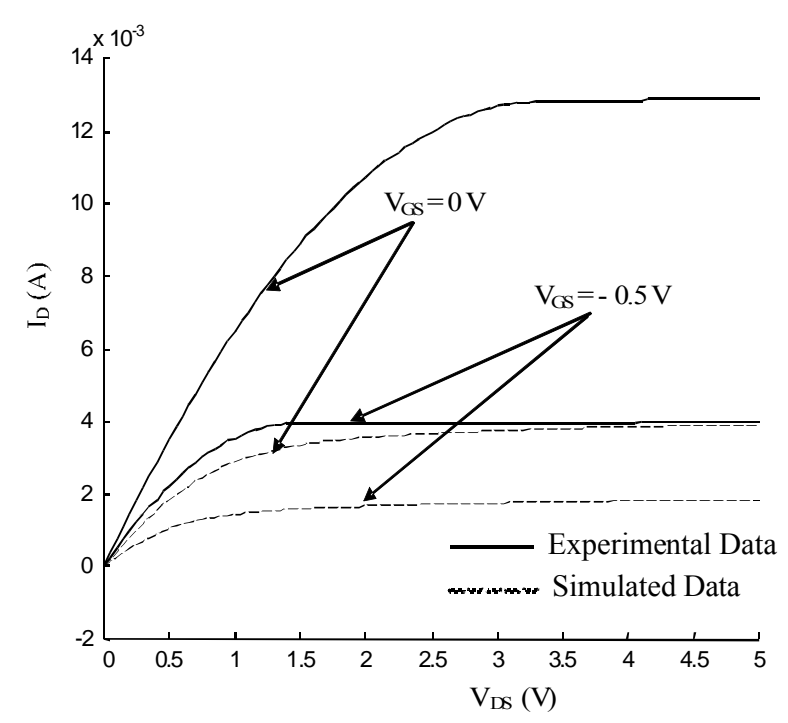

Fig. 10. Comparison between simulation data using default parameters and experimental data.

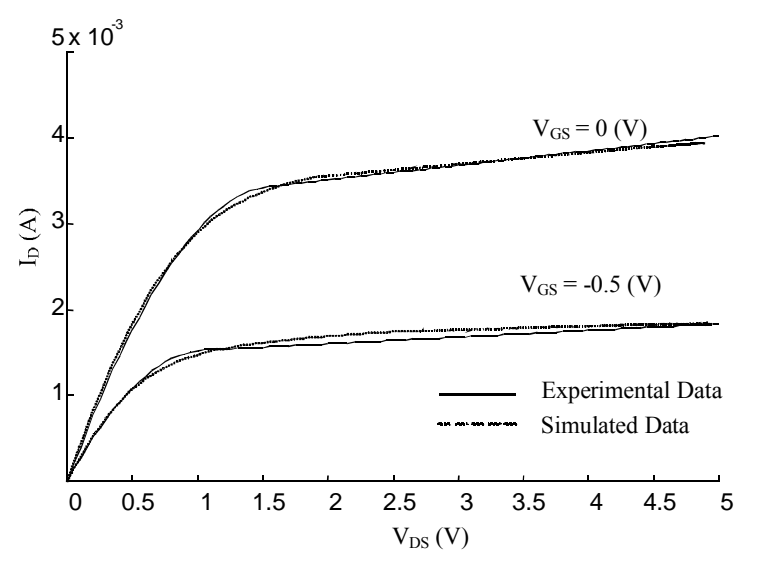

Fig. 11. Comparison between simulation data using the extracted parameters obtained by the optimisation method and experimental data.

Table 2. 2N3823 model parameters.

\begin{tabular}{cccc} 
Parameter & $\begin{array}{c}\text { Default } \\
(\text { Spice })\end{array}$ & $\begin{array}{c}\text { Extracted } \\
\text { (Graphical } \\
\text { method })\end{array}$ & $\begin{array}{c}\text { Extracted } \\
\text { (Optimization } \\
\text { method) }\end{array}$ \\
\hline$\beta\left(\mathrm{AV}^{-2}\right)$ & $1.17 \times 10^{-3}$ & $1.8925 \times 10^{-3}$ & $1.3627 \times 10^{-3}$ \\
$r_{D}(\mathrm{Ohm})$ & 1 & 2.34 & 2.34 \\
$r_{S}(\mathrm{Ohm})$ & 1 & 2.27 & 2.27 \\
$\lambda\left(\mathrm{V}^{-1}\right)$ & $4 \times 10^{-3}$ & $16.844 \times 10^{-3}$ & $54.155 \times 10^{-3}$ \\
$V_{T 0}(\mathrm{~V})$ & -3.3 & -1.443 & -1.533 \\
$I_{S}(\mathrm{fA})$ & 33.57 & 15.06 & 15.06 \\
$n$ & 1 & 1.1934 & 1.1934 \\
$C_{G D}(\mathrm{pF})$ & 1.6 & 4.59 & 4.59 \\
$C_{G S}(\mathrm{pF})$ & 2.414 & 4.62 & 4.62 \\
$m$ & 0.3622 & 0.7914 & 0.7914 \\
$\phi(\mathrm{V})$ & 1 & 1.0682 & 1.0682 \\
\hline
\end{tabular}

\subsection{Bipolar junction transistor testing}

The device that has been selected to present the developed approach is the BJT 2N2219A. The same approach used for testing the JFET has been also used for the above bipolar junction transistor. The simulation data consist of obtaining the collector current $\mathrm{I}_{\mathrm{C}}$ as a function of collector-emitter voltage $V_{C E}$ for different values of $I_{B}$ using default model parameters and then using the extracted values. These two sets of values are reported in Table 3.

Table 3. Default and extracted BJT model parameters.

\begin{tabular}{ccc} 
Parameter & Default & Extracted \\
\hline$I_{S}(\mathrm{fA})$ & 14.34 & 4.774 \\
$n_{F}$ & Not defined & 1.019 \\
$I_{K F}(\mathrm{~A})$ & $24.47 \times 10^{-2}$ & $4.015 \times 10^{-2}$ \\
$C_{2}$ & 1 & 6.43 \\
$n_{E L}$ & 1.307 & 2.05 \\
$\beta_{F}$ & 255.9 & 148.26 \\
$n_{R}$ & 0 & 1.012 \\
$I_{K R}(\mathrm{~A})$ & Not defined & $1.59 \times 10^{-2}$ \\
$C_{4}$ & 0 & 6.84 \\
$n_{C L}$ & 2 & 1.50 \\
$\beta_{R}$ & 6.092 & 3.78 \\
$V_{A}(\mathrm{~V})$ & 74.03 & 105.4 \\
$C_{B E}(0)(\mathrm{pF})$ & 22.01 & 23.34 \\
$C_{B C}(0)(\mathrm{pF})$ & 7.306 & 14.63 \\
$\phi_{E}(\mathrm{~V})$ & 0.75 & 0.77 \\
$m_{E}$ & 0.377 & 0.323 \\
$\phi_{C}(\mathrm{~V})$ & Not defined & 0.44 \\
$m_{C}$ & Not defined & 0.27 \\
\hline
\end{tabular}

The $I-V$ characteristics obtained for the $2 \mathrm{~N} 2219 \mathrm{~A}$ BJT using default device parameters, extracted parameters and experimental data are shown in Fig. 12. Note the improved accuracy of the characteristics of the curves based on extracted parameters as these generate $I$ $V$ curves that are very close to the experimental ones. The departure of the simulated $I-V$ characteristics with respect to the experimental curves can reach $30 \%$ for curves obtained using default values, and are only limited to less than $2.5 \%$ using extracted values.

From the obtained curves of Fig. 12, it appears that characteristics obtained using extracted parameters are better than those obtained using default parameters. But these characteristics are very accurate mainly due to the difficulty of calculating some devices parameters, especially those related to the drop of $\beta$ at low current levels $\left(C_{2}\right.$ and $\left.C_{4}\right)$. This difficulty is related to the presence of disturbing currents due to thermal noise effects which are not negligible at low current levels. Improved results can certainly be obtained from measurements at low temperatures within isothermal environment. In these conditions, the effect of disturbing currents can be highly reduced. 


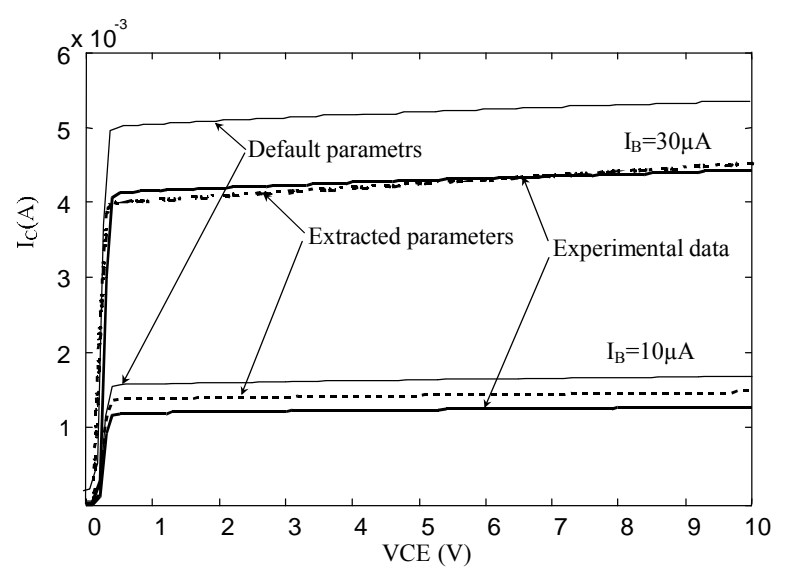

Fig. 12. Comparison between simulation data using the default parameters, extracted parameters and experimental data.

\section{Conclusion}

Data acquisition, parameter extraction and characterization integrated instrumentation system for electronic active components has been developed. Acquisition, monitoring and control tasks have been performed using highly sensitive measuring equipments and the extraction models implemented and based on established optimization techniques. The developed system has been tested on p-n junction and Schottky diodes, field effect transistors and bipolar junction transistors.

The performance of the developed extraction techniques was demonstrated by comparing experimental characteristics with Spice simulated curves using default parameters and model parameters extracted graphically or using optimization techniques. The relative excursions of the simulated $I-V$ characteristics of the most investigated devices were less than $2.5 \%$ with respect to the experimental curves, which shows the effectiveness of the developed system. In addition to the developed hardware, a number of software routines have also been implemented under Matlab environment to extract the Spice model parameters for the above devices. The developed system will be of great usefulness in industrial environments as well as for engineers in the characterization field of electronic devices.

\section{References}

1. P.R. Karlsson, Direct extraction of MOS transistor current model parameters // Technical Report No. 248, Department of Solid State Electronics, School of Electrical and Computer Engineering, Chalmers University of Technology, Göteborg; Sweden, 1996.

2. P.R. Karlsson, and K.O. Jeppson, An efficient parameter extraction algorithm for MOS transistor models // IEEE Trans. Electron Devices 39, No 9, pp. 2070-2076 (1992).

3. B. Beker, G. Cokkinides, M. Sechrest, Field, circuit, and visualization based simulation methodology for passive electronic components // Proc. 33rd Annual Simulation Symposium (SS 2000), 16-20 April, 2000, pp. 157-164.

4. K. Laouamri, J.-P. Keradec, J.-P. Ferrieux, J. Barbaroux, Magnetics, dielectric losses of capacitor and ferrite core in an LCT component // IEEE Trans. Magnetics 39, pp. 1574-1577 (2003).

5. Y. Wang, J. Callaway, T.P. Cass Chow, F. Wang, D. Boroyevich, SPICE Model of SiC JFETs for Circuit Simulations // IEEE Workshops on Computers in Power Electronics, COMPEL'06, 2006, pp. 212-215.

6. C. Buttay, H. Morel, B. Allard, P. Lefranc, O. Brevet, Model requirements for simulation of low-voltage MOSFET in automotive applications // IEEE Trans. Power Electronics 21(3), pp. 613-624 (2006).

7. J. Huijie, Yu Lai, X. Li, Y. Luo, L. Fursin, J.H. Zhao, P. Alexandrov, B. Wright, M. Weiner, An IGBT and MOSFET gated $\mathrm{SiC}$ bipolar junction transistor // 37th IAS Annual Meeting of the Industry Applications Conference, 4, 2002, pp. 2609-2613.

8. Hewlett Packard Characterization Solutions, A quarterly Publication of Semiconductor Systems Center, Winter 1992.

9. S.L. Wong and C.A.T. Salama, Improved Simulation of $\mathrm{p}$ - and n- channel MOSFET's using an enhanced SPICE MOS3 model // IEEE Trans. Computer-Aided Design, CAD-6, 4, pp. 586-591 (1987).

10. G.M. Buiatti, F. Cappelluti, G. Ghione, Finite difference based power diodes simulation within SPICE: Modeling approach and validation // IEEE 36th Power Electronics Specialists Conference, PESC'05, pp. 999-1003, 2005.

11. D.E. Ward and K. Doganis, Optimized extraction of MOS model parameters // IEEE Trans. Computer-Aided Design of Integrated Circuits and Systems, CAD-1, No 4, pp. 163-168 (1982).

12. F.M. Klaassen, W. de Groot, and F.L. van de Markt, Computer algorithm to determine MOS process-parameters // Philips Res. Repts. 31, pp. 84-92 (1976).

13. M.F. Hamer, First-order parameter extraction on enhancement silicon MOS transistors // IEE Proc. 133, Pt. I, 2, pp. 15-22 (1986).

14. H.P. Tuinhout, S. Swaving, and J.J.M. Joosten, A fully analytical MOSFET model parameter extraction approach // IEEE Proc. Microelectronic Test Structures, 1(1), pp. 79-84, (1988).

15. Paolo Antognetti and Giuseppe Massobrio, Semiconductor Device Modeling with SPICE. Mc Graw-Hill Book Company, 1988.

16. ORCAD Reference Manual.

17. R.M. Warner Jr., B.L. Grung, Transistors Fundamentals for the Integrated-Circuit Engineer. John Wiley and sons, Inc., 1983. 